\title{
Pronóstico de pacientes pediátricos con falla hepática aguda: estudio de cohorte
}

\author{
Prognostic of pediatric patients with acute liver failure: a cohort study \\ Omar Eugenio Naveda-Romero, ${ }^{*,+}$ Andrea Naveda-Meléndez, ${ }^{\ddagger}$ Rafael Meléndez-Freitez ${ }^{\ddagger}$ \\ * Hospital Universitario de Pediatría “Dr. Agustín Zubillaga”; ${ }^{\ddagger}$ Facultad de Ciencias de la Salud. Decanato de \\ Medicina. Universidad Centro-occidental “Lisandro Alvarado”. Barquisimeto, Estado Lara, Venezuela.
}

\begin{abstract}
RESUMEN
Introducción: La falla hepática aguda (FHA) es un síndrome multisistémico, rápidamente progresivo y con alta mortalidad. Objetivo: Determinar factores pronósticos de mala evolución en niños con FHA. Material y métodos: Estudio de una cohorte, retrospectiva, de niños menores de 14 años de edad. Los pacientes fueron agrupados según su pronóstico, favorable (recuperación) y desfavorable (muerte o necesidad de trasplante hepático [TH]) para comparar factores clínicos y de laboratorio. Para determinar la asociación de factores asociados a mal pronóstico se hizo mediante análisis de riesgo proporcionales de Cox. Resultados: Se incluyeron 44 pacientes. En la mayoría no se pudo identificar la etiología de la FHA (56.8\%). Quince pacientes (34.1\%) tuvieron evolución favorable, y $29(65.9 \%)$ mala evolución: 15 fallecieron y 14 cumplieron criterios de TH. En el modelo multivariado, los factores de mal pronóstico fueron: INR (razón normalizada internacional $)>3.5\left(\mathrm{HR}=2.235 ; \mathrm{IC}_{95} \%: 1.722-6.914 ; \mathrm{p}=\right.$ 0.043) y el mayor puntaje de LIU (Liver Injury Unit scoring system) $\left(\mathrm{HR}=2.0 ; \mathrm{IC}_{95} \%: 1.996-2.005 ; \mathrm{p}=0.049\right)$. Conclusiones: En niños con FHA la mortalidad es alta. Los factores asociados a una evolución desfavorable fueron INR $>3.5$ o puntajes LIU elevados.
\end{abstract}

Palabras clave: Falla hepática aguda, niños, pronóstico, mortalidad, trasplante hepático.

\begin{abstract}
Introduction: Acute liver failure (ALF) is a rapidly progressive and multisystem syndrome with high mortality. Objective: To determine prognostic factors of poor outcome in children with ALF. Material and methods: This is a retrospective cohort study with children under 14 years of age. In order to compare clinical and laboratory factors, patients were grouped according to their prognosis, favorable (recovery) and unfavorable (death or need for liver transplantation [LT]). Cox proportional risk analysis was used to determine the association of factors associated with prognosis. Results: 44 patients were included. In the majority, the etiology of ALF could not be identified (56.8\%). Fifteen patients (34.1\%) had a favorable evolution, and 29 (65.9\%) had a poor evolution: 15 died and 14 met $L T$ criteria. In the multivariate model, the poor prognostic factors were: INR (international normalized ratio) > 3.5 ( $H R=2.235 ; 95 \% \mathrm{Cl}: 1.722-6.914 ; p=0.043)$ and a higher LIU score (Liver Injury Unit scoring system) (HR = 2.0; 95\% Cl: 1.996-2.005; $p=0.049$ ). Conclusions: Mortality in children with ALF is high. Factors associated with an unfavorable evolution were INR $>3.5$ or high LIU scores.
\end{abstract}

Keywords: Acute liver failure, children, prognosis, mortality, liver transplantation.

\footnotetext{
+ Correspondencia: OENR, omarnavedamd@yahoo.com Conflicto de intereses: Los autores declaran que no tienen.

Citar como: Naveda-Romero OE, Naveda-Meléndez A, Meléndez-Freitez R. Pronóstico de pacientes pediátricos con falla hepática aguda: estudio de cohorte. Rev Mex Pediatr. 2020; 87(6); 212-217. https://dx.doi.org/10.35366/97682
} 


\section{INTRODUCCIÓN}

La falla hepática aguda (FHA) en niños es una enfermedad compleja, poco común, que puede evolucionar rápidamente a insuficiencia de las funciones del hígado. A pesar de los avances terapéuticos, esta situación resulta en muerte o trasplante hepático (TH) en más del $45 \%$ de los casos. ${ }^{1}$ Para la caracterización de la FHA se creó el grupo de estudio de la FHA pediátrica, empleando una nomenclatura estandarizada, la cual es ampliamente utilizada. ${ }^{2} \mathrm{~A}$ pesar de los avances en la terapéutica de esta entidad, la supervivencia sin trasplante continúa siendo baja. ${ }^{3}$ En Latinoamérica tanto la morbilidad como la mortalidad son elevados. ${ }^{4}$

Diversos factores se han estudiado sobre el pronóstico, tales como la edad, etiología de la FHA, presencia de encefalopatía, niveles séricos de bilirrubina, factores de coagulación (en particular, protrombina mediante INR [razón normalizada internacional]), amonio, lactato sérico, entre otros..$^{5,6}$ Con estos datos, se han desarrollado sistemas de puntuación que pueden ayudar a predecir el riesgo de fallecimiento o para la decisión de realizar TH. ${ }^{7}$ En la actualidad, la INR y el factor $\mathrm{V}$ son los mejores indicadores de mortalidad sin trasplante en niños con FHA. Uno de estos sistemas creado para pronosticar la evolución es el sistema de puntuación en unidades de daño hepático (Liver Injury Unit scoring system [LIU], por sus siglas en inglés), que incluye valores de bilirrubina, INR y amonio en el momento del ingreso, así como su evolución durante la hospitalización del paciente. ${ }^{8}$

El objetivo principal de este estudio fue determinar los factores pronóstico asociados a mortalidad y la necesidad de TH de pacientes pediátricos con FHA, y como objetivo secundario evaluar la utilidad del LIU para identificar la progresión del daño hepático a partir de la admisión hospitalaria.

\section{MATERIAL Y MÉTODOS}

Se llevó a cabo un estudio de cohorte, retrospectivo, mediante la revisión de expedientes de todos los pacientes menores de 14 años de edad con diagnóstico de FHA, admitidos en la Unidad de Cuidados Intensivos del Hospital Universitario de Pediatría "Dr. Agustín Zubillaga”, entre 2005 y 2019.

La FHA fue definida según los criterios del grupo de estudio de la FHA pediátrica. ${ }^{2}$ Se excluyeron aquellos pacientes que presentaron disfunción hepática crónica o con falla hepática secundaria a enfermedades sisté- micas, y quienes recibieron hemoderivados o vitamina $\mathrm{K}$ antes de su admisión.

Todos los pacientes fueron manejados de acuerdo a las recomendaciones del grupo de trabajo de la Sociedad Latinoamericana de Gastroenterología, Hepatología y Nutrición Pediátrica. ${ }^{9}$

Se evaluó la evolución de todos los pacientes incluidos durante su estancia hospitalaria. Como evolución favorable se consideró a los pacientes que tuvieron recuperación espontánea, o bien, si respondieron al tratamiento etiológico. Por el contrario, evolución desfavorable fue para pacientes que reunieron criterios para $\mathrm{TH}$ o fallecieron dentro de las 12 semanas desde su admisión. La estancia hospitalaria fue calculada hasta el egreso del paciente, su fallecimiento o hasta reunir criterios para $\mathrm{TH}$.

Las variables registradas fueron edad y sexo, etiología (indeterminada, infecciosa, vascular, cáncer, metabólica, tóxica), complicaciones (neurológicas, hemorrágicas, infecciosas, renales y respiratorias), datos de exámenes de laboratorio: transaminasa glutámicooxalacética (TGO), transaminasa glutámico-pirúvica (TGP), bilirrubina total, amonio, tiempo de protrombina (TP) e INR al momento del ingreso.

El diagnóstico de encefalopatía hepática se definió según los criterios de West Haven. Para la indicación de TH se utilizaron los criterios del King's College Hospital para insuficiencia hepática. ${ }^{10}$

Para el cálculo del puntaje LIU solamente se emplearon los datos de laboratorio al ingreso, mediante la siguiente fórmula: [3.507 $\times$ valor de bilirrubina total] + $[45.51 \times$ valor del INR $]+[0.254 \times$ valor del amonio $] .{ }^{11}$

\section{Análisis estadístico}

Las variables cuantitativas se presentan como promedio y desviación estándar; las variables cualitativas con frecuencias simples y porcentajes.

En la comparación entre los dos grupos (favorable o desfavorable) se utilizó $\chi^{2}$ de Pearson o prueba exacta de Fisher para los datos cualitativos y t de Student para datos cuantitativos. Se realizó análisis multivariante mediante regresión proporcionales de Cox; el modelo se construyó con las variables que en el análisis univariable tuvieron valor de $<0.1$, utilizado el método "introducir". Los resultados se expresaron como hazard ratio (HR) con un intervalo de confianza del 95\% (IC95\%).

Mediante curva ROC (receiver operating characteristic) y el índice de Youden se obtuvo el punto para obtener un punto de corte de la puntuación del puntaje LIU. El área bajo la curva (ABC) fue estimada en forma 
no paramétrica, con un intervalo de confianza del 95\%. Se construyeron curvas de supervivencia actuariales; la comparación fue con la prueba de Wilcoxon.

Se consideró significativa toda $\mathrm{p}<0.05$. Todos los análisis se realizaron con el programa SPSS ${ }^{\circledR}$, versión 17.0.

Aspectos éticos. El estudio fue aprobado por el Comité de Bioética de la institución; a fin de mantener la confidencialidad, se creó una base de datos codificada.

\section{RESULTADOS}

Se incluyeron 44 pacientes, la etiología más frecuente de FHA fue la indeterminada (56.8\%), seguida de la infecciosa (20.5\%) y vascular (9.1\%). Como se muestra en la Tabla 1, 15 pacientes (34.1\%) tuvieron evolución favorable, mientras que 29 (65.9\%) una mala evolución, de los cuales 15 fallecieron y 14 cumplieron criterios de $\mathrm{TH}$. Al comparar los factores entre los dos grupos se

Tabla 1: Comparación de las variables en 44 pacientes con falla hepática aguda, de acuerdo con su pronóstico.

\begin{tabular}{|c|c|c|c|}
\hline Variables & $\begin{array}{l}\text { Evolución favorable } \\
\qquad(n=15) \%\end{array}$ & $\begin{array}{l}\text { Evolución desfavorable } \\
\qquad(n=29) \%\end{array}$ & $\mathbf{p}^{\ddagger}$ \\
\hline Sexo masculino & 40.0 & 62.1 & 0.163 \\
\hline Edad (años)* & $6.0 \pm 3.9$ & $2.2 \pm 2.5$ & $<0.0001$ \\
\hline Edad $<2$ años & 33.3 & 69.0 & 0.024 \\
\hline Etiología & & & 0.609 \\
\hline Indeterminada & 53.3 & 58.6 & \\
\hline Infecciosa & 33.3 & 13.8 & \\
\hline Vascular & 6.7 & 10.3 & \\
\hline Oncohematológica & 6.7 & 6.9 & \\
\hline Metabólica & 0.0 & 6.9 & \\
\hline Tóxica & 0.0 & 3.4 & \\
\hline \multicolumn{4}{|l|}{ Complicaciones } \\
\hline Neurológica: encefalopatía & 33.3 & 62.1 & 0.070 \\
\hline Hemorrágicas & 60.0 & 44.8 & 0.340 \\
\hline Infecciosas & 26.7 & 51.7 & 0.112 \\
\hline Renales & 20.0 & 17.2 & 0.822 \\
\hline $\mathrm{SDER}^{* *}$ & 50.0 & 75.0 & 0.465 \\
\hline Respiratorias & 26.7 & 10.3 & 0.161 \\
\hline \multicolumn{4}{|l|}{ Datos de laboratorio } \\
\hline $\mathrm{TGO}(\mathrm{mg} / \mathrm{dL})^{*}$ & $1,389 \pm 348$ & $1,565 \pm 499$ & 0.221 \\
\hline $\operatorname{TGP}(\mathrm{mg} / \mathrm{dL})^{*}$ & $1,485 \pm 306$ & $1,511 \pm 423$ & 0.828 \\
\hline Bilirrubina total (mg/dL) & $18.1 \pm 3.0$ & $22.4 \pm 9.1$ & 0.084 \\
\hline Bilirrubina total > $17.5 \mathrm{mg} / \mathrm{dL}$ & 66.7 & 48.3 & 0.246 \\
\hline Amonio $(\mu \mathrm{g} / \mathrm{dL})^{*}$ & $131 \pm 38$ & $162 \pm 60$ & 0.082 \\
\hline Tiempo de protrombina* $(\mathrm{s})$ & $29 \pm 13$ & $42 \pm 26$ & 0.110 \\
\hline Tiempo de protrombina $>50(\mathrm{~s})$ & 20.0 & 37.9 & 0.226 \\
\hline $\mathrm{INR}^{*}$ & $2.6 \pm 1.1$ & $3.9 \pm 2.3$ & 0.048 \\
\hline INR $>3.5$ & 20.0 & 51.7 & 0.042 \\
\hline $\begin{array}{l}\text { Días de evolución antes del } \\
\text { ingreso* }\end{array}$ & $7.3 \pm 2.4$ & $7.8 \pm 3.1$ & 0.648 \\
\hline Puntaje LIU* & $215 \pm 57$ & $296 \pm 128$ & 0.023 \\
\hline Estancia hospitalaria (días)* & $32 \pm 12$ & $34 \pm 16$ & 0.601 \\
\hline
\end{tabular}

SDER = sistema de depuración extrarrenal; TGO = transaminasa glutámico-oxalacética; TGP = transaminasa glutámico-pirúvica; INR = razón normalizada internacional; LIU = Liver Injury Unit scoring system; $\mathrm{s}=$ segundos.

* Datos cuantitativos se expresan con promedio \pm desviación estándar.

** Con base en el total de ocho pacientes que presentaron complicaciones renales.

${ }^{\ddagger} \chi^{2}$ de Pearson o prueba de Fisher para datos cualitativos y t de Student 0 en los datos cuantitativos. 
Tabla 2: Modelo de regresión de Cox para identificar factores de riesgo de evolución desfavorable en niños con falla hepática aguda.

\begin{tabular}{lccc} 
Variables & HR & $\mathbf{I C}_{95 \%}$ & $\mathbf{P}$ \\
\hline Edad < 2 años & 2.077 & $0.853-5.058$ & 0.108 \\
Encefalopatía & 1.846 & $0.707-4.822$ & 0.211 \\
INR $>3.5$ & 2.235 & $1.722-6.914$ & 0.043 \\
Puntaje LIU & 2.000 & $1.996-2.005$ & 0.049 \\
\hline
\end{tabular}

$\mathrm{HR}=$ hazard ratios, IC = intervalo de confianza, LIU = Liver Injury Unit scoring system.

observó que, en general, no hubo diferencia. Sin embargo, el grupo con mala evolución presentó menor edad $(6.0 \pm 3.9$ vs $2.2 \pm 2.5$ años; $p<0.001)$, INR mayor de 3.5 (20.0 vs $51.7 \%$; $\mathrm{p}=0.042)$ y mayor puntaje de LIU ( $215 \pm 57$ vs $296 \pm 128$ puntos; $p=0.023)$. Un aspecto a destacar es que también hubo mayor proporción de pacientes con encefalopatía entre quienes tuvieron mala evolución; sin embargo, no fue estadísticamente significativo ( $62.1 \%$ vs $33.3 \%$; $=0.070)$.

Posteriormente, en el modelo multivariado (Tabla 2) se estableció que los factores predictores independientes asociados a un desenlace desfavorable fueron: $\mathrm{INR}>3.5\left(\mathrm{HR}=2.235 ; \mathrm{IC}_{95 \%}: 1.722-6.914 ; \mathrm{p}\right.$ $=0.043)$ y puntaje LIU $\left(\mathrm{HR}=2.0 ; \mathrm{IC}_{95 \%}: 1.996-2.005\right.$; $\mathrm{p}=0.049)$.

El punto de corte del puntaje LIU para identificar un mal desenlace mediante curva ROC se estableció en 240 puntos (ABC: 0.740; $\mathrm{IC}_{95 \%}: 0.592-0.889 ; \mathrm{p}=$ 0.010). En este valor, se obtuvo el mayor valor de índice de Youden (0.56), con una especificidad de $80 \%$ y una sensibilidad de $76 \%$ para predecir una evolución desfavorable en niños con FHA (Figura 1).

En la Figura 2 se muestran las curvas de supervivencia actuariales según el INR y el puntaje LIU. Al igual que los análisis previos, se observa que en los niños con INR $>3.5$ tuvieron menor supervivencia $(p=0.039)$, y que puntajes de LIU $\geq 240$ también se asociaron a mal pronóstico $(\mathrm{p}=0.048)$.

\section{DISCUSIÓN}

En este estudio de niños con FHA se pudo comprobar que tienen una tasa elevada de fallecimiento (34.1\%), pero además se pudo identificar que hay factores, como el INR y puntaje de LIU, que pueden ayudar a predecir la evolución tanto para la necesidad de TH como de mortalidad.
En general, las características de la población estudiada resultaron similares a estudios previos, incluyendo que su etiología en la mayoría de los casos no se pudo determinar. ${ }^{12,13}$

Asimismo, como en otros estudios, los pacientes de menor edad, tuvieron mayor riesgo de tener evolución desfavorable. ${ }^{14}$ Es posible que la menor edad, por disminución de la opsonización, quimiotactismo y baja activación del sistema de complemento, aumente la susceptibilidad a procesos infecciosos, lo cual es causa importante de muerte en pacientes con FHA. Por otra parte, las complicaciones neurológicas son las que con mayor frecuencia determinan la progresión de los pacientes con FHA. ${ }^{15}$ En este estudio, la encefalopatía hepática estuvo asociada a la evolución adversa, lo que coincide con lo reportado en la literatura, ${ }^{16}$ debido a hipertensión endocraneana secundaria a edema cerebral. No obstante, la edad ni la encefalopatía se identificaron como factores independientes de mal pronóstico (Tabla 2).

En concordancia con lo reportado en estudios previos, ${ }^{17,18}$ la INR constituyó un factor predictor independiente de mala evolución. Los trastornos de coagulación son un componente común en los pacientes con FHA y predisponen a sangrado. El trastorno en la cascada de coagulación se refleja en la prolongación del TP, por disminución en la producción de los factores: I, II, V, VII, IX y X.

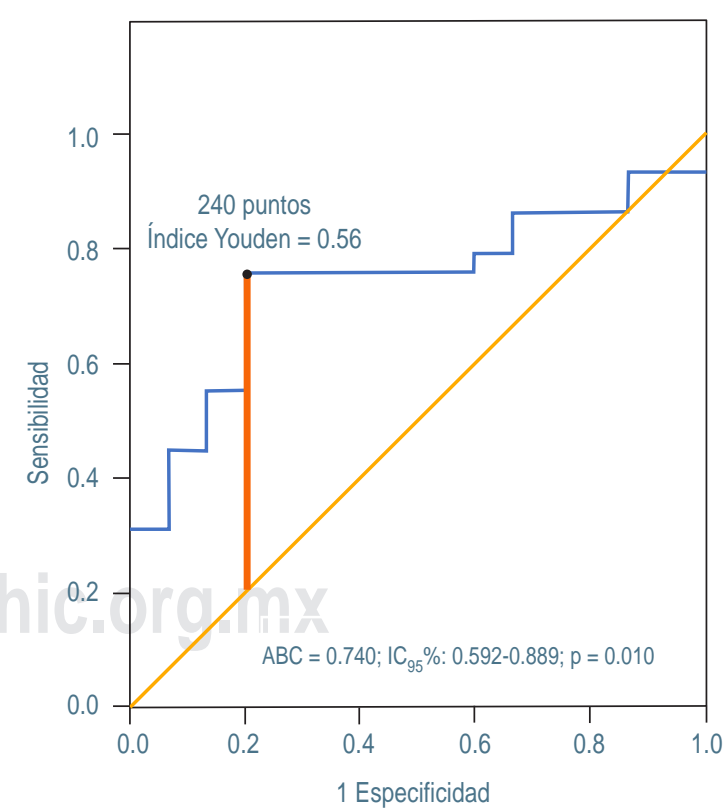

Figura 1: Curva ROC para el puntaje LIU en niños con falla hepática aguda. 


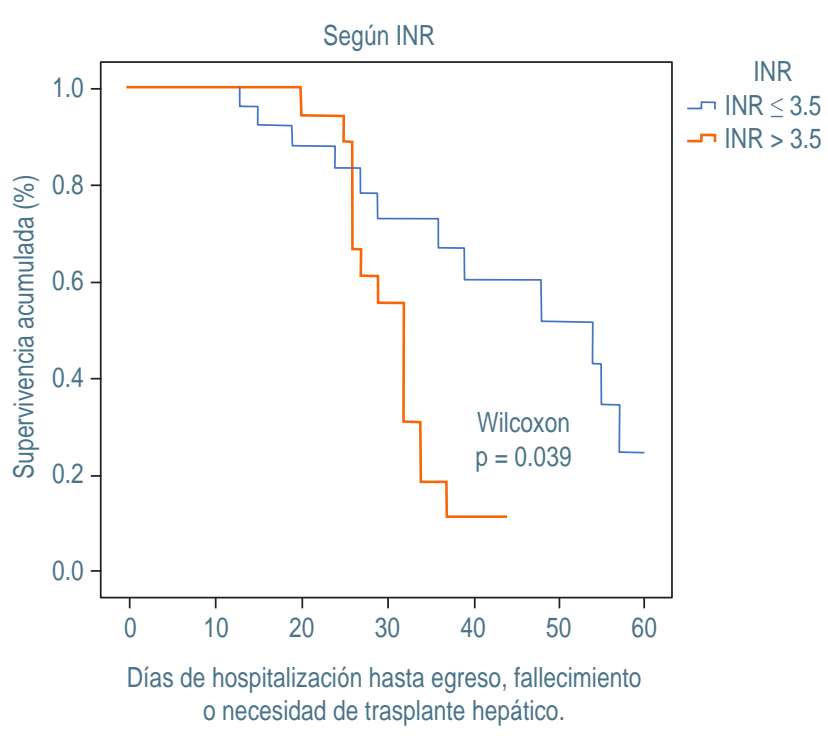

Figura 2: Curvas de supervivencia actuarial.

Para determinar las condiciones de gravedad al momento de ingreso de los niños con FHA, se utilizan los criterios del grupo del King's College Hospital; sin embargo, sus resultados no han sido homogéneos en niños. ${ }^{19}$ En el presente estudio comprobamos que el puntaje LIU podría contribuir en la toma de decisiones en relación a la indicación y el momento oportuno del TH en niños. ${ }^{4}$ Esta herramienta incluye los valores de bilirrubina total, INR y amonio; cuando se realizaron los diferentes análisis se identificó que puntajes $>240$ puntos tienen una especificidad del $80 \%$ y una sensibilidad del 76\% para pronosticar una evolución adversa, los cuales son resultados semejantes a otros estudios. ${ }^{7,11}$

La mortalidad de la FHA en la edad pediátrica varía entre 30 y $70 \% .^{4}$ En esta cohorte, la mortalidad fue de $34.1 \%$; sin embargo, es conveniente mencionar que fallecieron la mitad de los pacientes que cumplieron con los criterios para $\mathrm{TH}$ antes de que pudiera realizarse este procedimiento. Debido a las limitaciones propias de países en vías de desarrollo, como los costos económicos, las dificultades en la selección de donantes y el tipo de donante hacen poco factible que se realice un trasplante. En este contexto, el sistema de diálisis con albúmina MARS (Molecular Adsorbent Recirculating System), técnica de hemodiafiltración extracorpórea, que a pesar de no incrementar la supervivencia de los pacientes con FHA, podría constituir un enlace hacia el TH, manteniendo al paciente en condiciones ideales hasta que exista la posibilidad de un trasplante o se recuperen las funciones del hígado nativo.

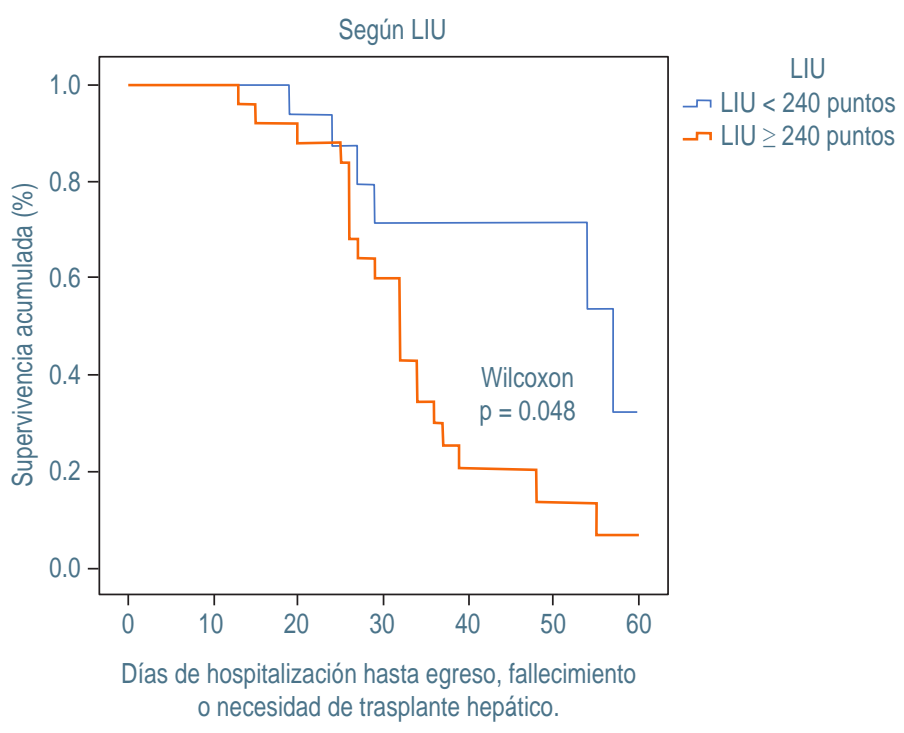

\section{Limitaciones del estudio}

Es importante destacar que el número de pacientes incluidos fue reducido. Además, no se investigaron algunos biomarcadores que potencialmente podrían estar relacionados con la evolución, como: alfa-fetoproteína, fósforo y lactato sérico. Por la naturaleza retrospectiva del estudio, tampoco se pudieron evaluar otras escalas de mortalidad pediátrica, como PRISM III o PELOD. Además, debido a que nuestro hospital no cuenta con un programa de trasplante, no se utilizó la escala PELD (Pediatric Model for End-Stage Liver Disease).

\section{REFERENCIAS}

1. Oh SH, Kim KM, Kim DY, Kim Y, Song SM, Lee YJ et al. Improved outcomes in liver transplantation in children with acute liver failure. J Pediatr Gastroenterol Nutr. 2014; 58: 68-73.

2. Narkewicz MR, Dell Olio D, Karpen SJ, Murray KF, Schwarz K, Yazigi N et al; Pediatric Acute Liver Failure Study Group. Pattern of diagnostic evaluation for the causes of pediatric acute liver failure: an opportunity for quality improvement. J Pediatr. 2009; 155: 801-806.

3. Mahadeb P, Grass J, Sokal E, Otte JB, Lerut J, Detaille T et al. Liver transplantation in children with fulminant hepatic failure: the UCL experience. Pediatr Transplant. 2009; 13: 414-420.

4. Reyes-Cerecedo A, Flores-Calderón J, Villasis-Keever MA, OrtizGalván RC, Jean-Aurelus P, Cuervo-Moreno E et al. Consenso para el manejo de la falla hepática aguda en pediatría. Rev Mex Pediatr. 2017; 84(3): 120-128.

5. Ciocca M, Ramonet M, Cuarterolo M, López S, Cernadas C, Álvarez F. Prognostic factors in paediatric acute liver failure. Arch Dis Child. 2008; 93 (1): 48-51. 
6. Gilbert Pérez JJ, Jordano Moreno B, Rodríguez Salas M. Etiología, resultados e indicadores pronósticos del fallo hepático agudo pediátrico. An Pediatr (Barc). 2018; 88: 63-68.

7. Liu E, MacKenzie T, Dobyns EL, Parikh CR, Karrer FM, Narkewicz MR et al. Characterization of acute liver failure and development of a continuous risk of death staging system in children. $J$ Hepatol. 2006; 44: 134-141.

8. Lu BR, Zhang S, Narkewicz MR, Belle SH, Squires RH, Sokol RJ et al. Evaluation of the liver injury unit scoring system to predict survival in a multinational study of pediatric acute liver failure. $J$ Pediatr. 2013; 162: 1010-1016.

9. Ciocca M, Costagua A, Cuarterolo M, Delgado L, Garcete L, Godoy M et al. Insuficiencia hepática aguda pediátrica. Grupo de trabajo de la Sociedad Latinoamericana de Gastroenterología, Hepatología y Nutrición Pediátrica (SLAGHNP). Acta Gastroenterol Latinoam. 2016; 46: 52-70.

10. Dhiman RK, Jain S, Maheshwari U, Bhalla A, Sharma N, Ahluwalia $\mathrm{J}$ et al. Early indicators of prognosis in fulminant hepatic failure: an assessment of the Model for End-Stage Liver Disease (MELD) and King's College Hospital Criteria. Liver Transpl. 2007; 13: 814-821.

11. Lu B, Gralla J, Liu E, Dobyns E, Narkewicz M, Sokol R. Evaluation of a scoring system for assessing prognosis in pediatric acute liver failure. Clin Gastroenterol Hepatol. 2008; 6(10): 1140-1145.
12. Devictor $D$, Tissieres $P$, Afanetti M, Debray D. Acute liver failure in children. Clin Res Hepatol and Gastroenterol. 2011; 35: 430-437.

13. Jimenez JM, Polo B, Donat E. Fallo hepático agudo. Protocolos diagnóstico-terapéuticos de Gastroenterología, Hepatología y Nutrición Pediátrica SEGHNP-AEP. Tema 3. pp. 197-204.

14. Sundaram S, Alonso E, Narkewicz M, Zhang S, Squires RH; Pediatric Acute Liver Failure Study Group. Characterization and outcomes of young infants with acute liver failure. J Pediatr. 2011; 159: 813-818.

15. Castillo L, Pérez C, Ruiz C, Bugedo G, Hernández G, Martínez J et al. Intravascular hypothermia for the management of intracranial hypertension in acute liver failure: case report. Rev Med Chil. 2009; 137: 801-806.

16. Squires R. Expanded neurologic assessment in pediatric acute liver failure: an important initial step. J Pediatr Gastroenterol Nutr. 2014; 58: 394-395.

17. Squires RHJR, Shneider BL, Bucuvalas J, Alonso E, Sokol RJ, Narkewicz MR et al. Acute liver failure in children: The first 348 patients in the pediatric acute liver failure study group. J Pediatr. 2006; 148: 652-658.

18. Kaur S, Kumar P, Kumar V, Sarin SK, Kumar A. Etiology and prognostic factors of acute liver failure in children. Indian Pediatr. 2013; 50: 677-679.

19. Yantorno SE, Kremers WK, Ruf AE. MELD is superior to King's College and Clichy's Criteria to assess prognosis in fulminant hepatic failure. Liver Transpl. 2007; 13: 822-828. 